\title{
RANKING OF WEIGHTED MAJORITY RULES
}

\author{
DANIEL BEREND, ${ }^{*}$ \\ YURI CHERNYAVSKY ${ }^{* *}$ AND \\ LUBA SAPIR, ${ }^{* * *}$ Ben-Gurion University
}

\begin{abstract}
A decision-making body may utilize a wide variety of different strategies when required to make a collective decision. In principle, we would like to use the most effective decision rule, that is, the rule yielding the highest probability of making the correct decision. However, in reality we often have to choose a decision rule out of some restricted family of rules. Therefore, it is important to be able to rank various families of rules. In this paper we consider three classes of decision rules: (i) balanced expert rules, (ii) the so-called single expert rules, and (iii) restricted majority rules. For the first two classes, we show that, as we deviate from the best rule in the family, the effectiveness of the decision rule decreases. For the last class, we obtain a very different phenomenon: any inner ranking is possible.
\end{abstract}

Keywords: Decision rule; weighted majority rule; balanced expert rule; restricted majority rule; dichotomous choice model

2000 Mathematics Subject Classification: Primary 91B06; 91B02; 91B12

\section{Introduction}

There are many situations in which an important decision is to be taken by a panel of experts, who are expected to have sufficient knowledge of the subject so that their decision will be more accurate than random guessing. Examples include boards of directors, surgical teams, and admissions committees. We concentrate on the uncertain dichotomous choice model, which goes back as far as Condorcet (1785). In this model, a group of $n$ decision makers is required to select one of two alternatives, only one of which is correct. We assume that the alternatives are symmetric. Namely, the a priori probabilities of the alternatives are equal, and the benefit or loss associated with a correct or incorrect decision, respectively, is the same in both cases. Each expert $i, 1 \leq i \leq n$, selects independently of the others and has his own correctness probability $p_{i}$, indicating his ability to identify the correct alternative. It is also assumed that $\frac{1}{2} \leq p_{i}<1,1 \leq i \leq n$, and, with no loss of generality, that $p_{i} \geq p_{j}, 1 \leq i<j \leq n$. The correctness probabilities are independent of which alternative is the correct one (cf. Nitzan and Paroush (1985)). A decision rule translates the individual opinions of the members, or a voting profile, into a group decision. A decision rule $v$ is optimal for a group of experts with correctness probabilities $\boldsymbol{p}=\left(p_{1}, p_{2}, \ldots, p_{n}\right)$ if it maximizes the effectiveness $\pi(v, \boldsymbol{p})$, namely, the likelihood of the group to make a correct choice.

\footnotetext{
Received 3 April 2008; revision received 1 September 2008.

* Postal address: Departments of Mathematics and of Computer Science, Ben-Gurion University, Beer-Sheva 84105, Israel. Email address: berend@cs.bgu.ac.il

** Postal address: Department of Computer Science, Ben-Gurion University, Beer-Sheva 84105, Israel.

Email address: yurac@cs.bgu.ac.il

*** Postal address: Department of Industrial Engineering and Management, Ben-Gurion University, Beer-Sheva 84105, Israel. Email address: 1sapir@bgumail.bgu.ac.il
} 
Nitzan and Paroush (1982), (1984), (1985, p. 15) obtained a criterion to identify the optimal decision rule for known values of correctness probabilities. They proved that the optimal decision rule is always a weighted majority rule. Specifically, for a committee of $n$ experts, the optimal rule is given by the vector of weights $\left(w_{1}, w_{2}, \ldots, w_{n}\right)$, where

$$
w_{i}=\log \frac{p_{i}}{1-p_{i}}, \quad 1 \leq i \leq n .
$$

The class of weighted majority rules contains the expert and simple majority rules as extremes. The expert rule assigns zero weights to all members of the group but the most qualified member, so that the group always follows his decision. The simple majority rule assigns equal weights to all decision makers, so that the group always follows the majority opinion.

A general comprehensive study of weighted majority rules is a complicated task, since the class of such rules becomes very large as the number of group members increases (cf. von Neumann and Morgenstern (1944), Isbell (1959), Muroga et al. (1967), Karotkin (1993), Karotin and Schaps (2003), and Berend and Sapir (2003)). For any group size, Karotkin (1998) showed how all the weighted majority rules can be arranged in a graph, where the nodes are the rules and the edges represent distinguishing voting profiles, namely, profiles on which corresponding rules disagree.

In this paper, for a fixed group size, we identify all possible rankings of the rules of several specific types. We are concerned with three families of rules: (i) balanced expert rules, (ii) single expert rules, and (iii) restricted majority rules. These rules will be defined rigorously in Section 2.

In the first and the last families, the rules are determined by the number of group members having an influence on the group decision. However, under the restricted majority rules, each of these members is equally influential, while the balanced expert rule gives the top member almost all the power, and he is outvoted only if opposed by all other influential members. In contrast, in the second family, all decision makers have an influence on the group decision. More precisely, the rules are determined by the weight assigned to the most competent expert, while all other experts are equally influential.

For each committee size, we arrange the rules in each of the three families of rules mentioned above in a sequence, ordered in a 'natural' way (e.g. the restricted majority rules are ordered by the number of influential experts).

The families of the balanced expert and the restricted majority rules were studied by Gradstein and Nitzan (1986), where an efficient optimality test for these rules was proposed for committees with known competence levels. Berend and Sapir (2003) computed the probability of these rules being optimal under partial information about the competence levels. Sapir (2005) obtained a criterion for ranking pairs of adjacent restricted majority rules (i.e. with numbers of influential experts differing by 2).

We prove that, for each committee size, in each of the sequences of single expert rules and of balanced expert rules, the likelihood of selecting the correct alternative is mound-shaped as a function of the index of the rule in the sequence. That is, the value of the likelihood function decreases as the parameter deviates from that of the most effective rule in the sequence, in both directions. In contrast, the family of restricted majority rules does not share this property. Moreover, for any group size, we prove that any ranking of the set of restricted majority rules may be realized.

The rest of this paper is organized as follows. In Section 2 we give precise definitions of the families of rules mentioned above and describe some of their properties. In Section 3 we rank all weighted majority rules for committees of five members and provide some motivation for 
our results for committees of arbitrary size. Section 4 contains the main results, and Section 5 is devoted to the proofs. We summarize and conclude the paper in Section 6.

\section{Weighted majority rule families}

Let us first formally define the families of weighted majority rules we investigate.

Definition 1. The balanced expert rule of order $k$, where $3 \leq k \leq n$, is denoted by $\mathrm{BER}_{n, k}$ and characterized by assigning weight of $k-2$ to the most competent expert, weight 1 to each of the $k-1$ next experts, and zero weight to the remaining $n-k$ experts. Namely, the rule is given by the vector

$$
(k-2, \underbrace{1, \ldots, 1}_{k-1}, \underbrace{0, \ldots, 0}_{n-k}) .
$$

Definition 2. Let $n, k$ be of the same parity, where $1 \leq k \leq n$. The single expert rule of order $k$ is denoted by $\mathrm{SER}_{n, k}$ and given by the vector

$$
(k, \underbrace{1, \ldots, 1}_{n-1}) .
$$

Definition 3. Let $k=2 s+1$, where $1 \leq s<n / 2$. The restricted majority rule of order $k$ is denoted by $\mathrm{RMR}_{k}$ and is equivalent to the simple majority rule, applied to the subgroup of the $k$ most competent experts. Namely, the rule is given by the vector

$$
(\underbrace{1, \ldots, 1}_{k}, \underbrace{0, \ldots, 0}_{n-k}) .
$$

For a committee of $n$ experts, the competence structure is defined either by a vector of correctness probabilities $\left(p_{1}, p_{2}, \ldots, p_{n}\right)$ or, equivalently, by a vector of expertise levels $\left(\alpha_{1}, \alpha_{2}, \ldots, \alpha_{n}\right)$, where $\alpha_{i}=p_{i} / q_{i}$ and $q_{i}=1-p_{i}$ for $1 \leq i \leq n$.

Let us now define the criterion for ranking rules. For a group of experts with competence structure $\boldsymbol{p}$, we define a weak order ' $\preccurlyeq$ ' between decision rules by $f \preccurlyeq g$ if $\pi(f, \boldsymbol{p}) \leq \pi(g, \boldsymbol{p})$. For convenience, we also use the notation ' $\prec$ ', ' $\succcurlyeq$ ', ' $\succ$ ', and ' $\sim$ ' (the latter being an equivalence relation).

We employ the method introduced by Sapir (2005) for ranking pairs of adjacent restricted majority rules. This method is based on the following notion.

Definition 4. Let $\boldsymbol{a}=\left(a_{1}, a_{2}, \ldots, a_{2 k+1}\right) \in \mathbb{R}^{2 k+1}$. The central mean of $\boldsymbol{a}$ is given by

$$
\operatorname{CM}(\boldsymbol{a})=\frac{\sigma_{k+1}(\boldsymbol{a})}{\sigma_{k}(\boldsymbol{a})},
$$

where

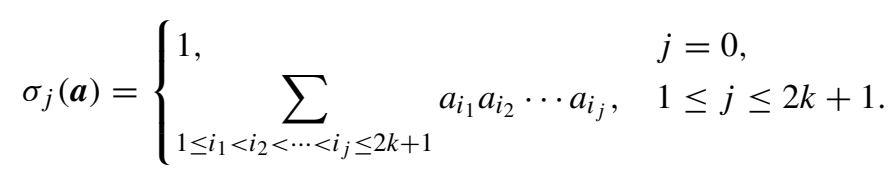

In the next sections we will use the following theorem and lemma, based on Sapir (2005).

Theorem 1. For each vector of expertise levels $\boldsymbol{\alpha}=\left(\alpha_{1}, \alpha_{2}, \ldots, \alpha_{n}\right)$ and odd $k, 1 \leq k \leq$ $n-2$,

1. $\mathrm{RMR}_{k} \succ \mathrm{RMR}_{k+2}$ if and only if $\alpha_{k+1} \alpha_{k+2}>\mathrm{CM}\left(\left(\alpha_{1}, \alpha_{2}, \ldots, \alpha_{k}\right)\right)$,

2. $\mathrm{RMR}_{k} \sim \mathrm{RMR}_{k+2}$ if and only if $\alpha_{k+1} \alpha_{k+2}=\mathrm{CM}\left(\left(\alpha_{1}, \alpha_{2}, \ldots, \alpha_{k}\right)\right)$. 
The first part of the theorem is proved in Sapir (2005). A slight modification of this proof gives the second part of the theorem.

Lemma 1. Let $\boldsymbol{a}=\left(a_{1}, \ldots, a_{n}\right)$, where $n \geq 3$ is odd and $a_{1} \geq a_{2} \geq \cdots \geq a_{n}>0$. Set $\boldsymbol{b}=\left(a_{1}, \ldots, a_{n-2}\right)$. Then, $\operatorname{CM}(\boldsymbol{a}) \leq \mathrm{CM}(\boldsymbol{b})$, with equality if and only if $a_{1}=a_{2}=\cdots=a_{n}$.

The inequality $\operatorname{CM}(\boldsymbol{a}) \leq \mathrm{CM}(\boldsymbol{b})$ is the contents of Lemma 1 of Sapir (2005). Going over the proof there, it is easy to establish that we have an equality if and only if $a_{1}=a_{2}=\cdots=a_{n}$.

\section{Motivation and illustration}

Consider the set of all weighted majority rules for a committee size of $n=5$, namely, the rules $(1,0,0,0,0),(3,1,1,1,1),(2,1,1,1,0),(3,2,2,1,1),(2,2,1,1,1),(1,1,1,1,1)$, and $(1,1,1,0,0)$. (In fact, any other vector of weights is equivalent to one of the above; see Nitzan and Paroush (1985, p. 21).)

The motivation to our present study goes back to Karotkin et al. (1988) and Karotkin (1998). It was shown there that the above rules can be arranged in the undirected graph depicted in Figure 1, where each node represents a rule and each edge corresponds to a pair of symmetric distinguishing voting profiles. For instance, the voting profiles $(+1,-1,-1,-1,-1)$ and $(-1,+1,+1,+1,+1)$ distinguish between the rules $(1,0,0,0,0)$ and $(3,1,1,1,1)$. Note that any two rules are distinguished by some set of voting profiles. In the graph, however, rules are adjacent only if they are distinguished by a single pair of symmetric voting profiles.

For each particular committee competence structure, Karotkin (1998) constructed a corresponding directed graph, where all edges are directed towards the endpoint rule of lower expected utility. For example, the directed graph obtained from the competence vector $(0.87$, $0.84,0.63,0.57,0.56)$ is depicted in Figure $2(a)$ and the one obtained from the competence vector $(0.97,0.72,0.64,0.64,0.55)$ is depicted in Figure $2(\mathrm{~b})$. It was shown that there exists a directed path from the node corresponding to the optimal rule to any other node. We refer the reader to Karotkin (1998) for more details.

Suppose that, for a certain committee, $(1,0,0,0,0)$ is the optimal rule. From the result of Karotkin, it follows that

$$
(3,2,2,1,1) \succcurlyeq(1,1,1,0,0) \text { and }(3,2,2,1,1) \succcurlyeq(2,2,1,1,1) \text {. }
$$

However, it does not specify which of the rules $(1,1,1,0,0)$ or $(2,2,1,1,1)$ is preferable. In fact, for this committee, there are at most three possible rankings among all rules. Namely,

$$
\begin{aligned}
& \text { 1. }(1,0,0,0,0) \succ(3,1,1,1,1) \succ(2,1,1,1,0) \succ(3,2,2,1,1) \succ(1,1,1,0,0) \succ \\
& \quad(2,2,1,1,1) \succ(1,1,1,1,1) ; \\
& \text { 2. }(1,0,0,0,0) \succ(3,1,1,1,1) \succ(2,1,1,1,0) \succ(3,2,2,1,1) \succ(2,2,1,1,1) \succ \\
& \quad(1,1,1,0,0) \succ(1,1,1,1,1) ; \\
& \text { 3. }(1,0,0,0,0) \succ(3,1,1,1,1) \succ(2,1,1,1,0) \succ(3,2,2,1,1) \succ(2,2,1,1,1) \succ \\
& \quad(1,1,1,1,1) \succ(1,1,1,0,0) .
\end{aligned}
$$

We verified that each of the above rankings can indeed be realized. This was done with the aid of a computer program which generates a large number of random competence vectors and tests the ranking for each of these. For example, ranking 2 above is obtained for the competence vector $(0.95,0.72,0.67,0.63,0.61)$. 


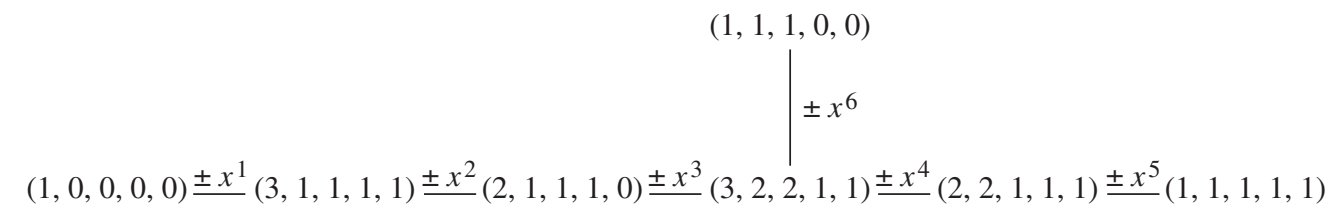

FIGURE 1: Graphs of weighted majority rules for $n=5$, where $x^{1}=(+1,-1,-1,-1,-1)$, $x^{2}=(+1,-1,-1,-1,+1), x^{3}=(+1,-1,-1,+1,-1), x^{4}=(+1,-1,+1,-1,-1)$, $x^{5}=(+1,+1,-1,-1,-1)$, and $x^{6}=(+1,-1,-1,+1,+1)$.

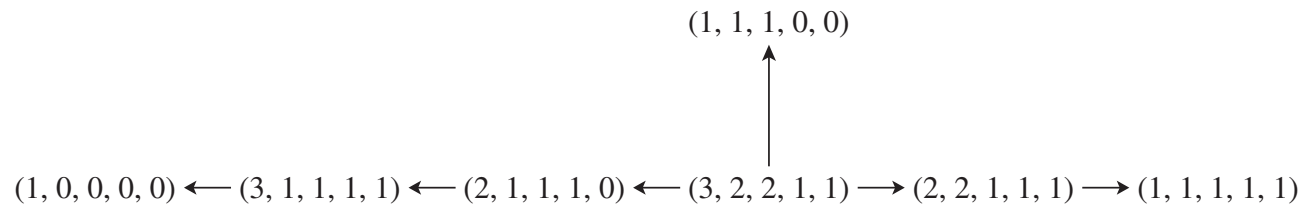

(a)

$(1,1,1,0,0)$

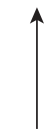

$(1,0,0,0,0) \longrightarrow(3,1,1,1,1) \longrightarrow(2,1,1,1,0) \longrightarrow(3,2,2,1,1) \longrightarrow(2,2,1,1,1) \longrightarrow(1,1,1,1,1)$

(b)

FIGURE 2: Various directed graphs of weighted majority rules for $n=5$ and competence vector (a) $(0.87,0.84,0.63,0.57,0.56)$, (b) $(0.97,0.72,0.64,0.64,0.55)$.

Similar analysis was performed in the case of other optimal rules. Table 1 summarizes our results. The second column provides the number of rankings, which are consistent with Figure 1. For example, if the rule $(3,2,2,1,1)$ is optimal then there are $\left(\begin{array}{c}6 \\ 1,2,3\end{array}\right)=60$ ways to order the six other rules. The third column gives the number of rankings that were actually observed. Thus, these columns provide upper and lower bounds on the number of possible rankings among all rules.

TABLE 1: Number of rankings among all rules.

\begin{tabular}{ccc}
\hline Optimal rule & Graph consistent & Observed \\
\hline$(1,0,0,0,0)$ & 3 & 3 \\
$(3,1,1,1,1)$ & 18 & 12 \\
$(2,1,1,1,0)$ & 45 & 29 \\
$(3,2,2,1,1)$ & 60 & 45 \\
$(1,1,1,0,0)$ & 10 & 10 \\
$(2,2,1,1,1)$ & 24 & 18 \\
$(1,1,1,1,1)$ & 4 & 3 \\
\hline Total & 164 & 120 \\
\hline
\end{tabular}


The following proposition shows that there exist rankings that cannot be realized even though they are not eliminated by the graph analysis performed above.

Proposition 1. 1. If $(3,1,1,1,1) \succ(2,1,1,1,0)$ then $(1,0,0,0,0) \succ(1,1,1,0,0)$.

2. If $(2,2,1,1,1) \succ(3,2,2,1,1)$ then $(1,1,1,0,0) \succ(1,0,0,0,0)$.

3. If $(3,2,2,1,1) \succ(2,1,1,1,0)$ then $(1,1,1,0,0) \succ(1,0,0,0,0)$.

4. $(3,1,1,1,1) \succ(3,2,2,1,1)$ if and only if $(1,0,0,0,0) \succ(1,1,1,0,0)$.

Proof. We will prove the first part. The rest of the proposition can be easily proved using similar arguments. Observe that $(3,1,1,1,1) \succ(2,1,1,1,0)$ implies that $p_{1} q_{2} q_{3} q_{4} p_{5}>$ $q_{1} p_{2} p_{3} p_{4} q_{5}$, or, equivalently, $\alpha_{1} \alpha_{5}>\alpha_{2} \alpha_{3} \alpha_{4}$. Since $\alpha_{4} \geq \alpha_{5}$, we have $\alpha_{1} \alpha_{5}>\alpha_{2} \alpha_{3} \alpha_{4} \geq$ $\alpha_{2} \alpha_{3} \alpha_{5}$ and $\alpha_{1}>\alpha_{2} \alpha_{3}$. From Theorem 1, it follows that $(1,0,0,0,0) \succ(1,1,1,0,0)$.

It follows from Proposition 1 that the empirical results in the third column of Table 1 are in fact the precise numbers of actual possibilities. For instance, suppose that $(1,1,1,1,1)$ is the optimal rule. The following ranking is impossible by part 3 of the proposition: $(1,1,1,1,1) \succ$ $(2,2,1,1,1) \succ(3,2,2,1,1) \succ(2,1,1,1,0) \succ(3,1,1,1,1) \succ(1,0,0,0,0) \succ(1,1,1,0,0)$.

In Appendix A we provide all 120 possible rankings for group size $n=5$.

The above discussion shows that there exists interesting information regarding possible rankings of weighted majority rules, which does not follow from the structure of the graph of Karotkin (1998). In the following two sections we consider decision bodies of arbitrary sizes. In the general case, the identification of all possible rankings between all rules becomes a formidable task. We focus on several special families of rules and identify all possible rankings within each family.

\section{Main results}

Theorem 2. For each $n$ and $\boldsymbol{p}$, the sequence of decision rules $\mathrm{BER}_{n, k}$ is mound-shaped as a function of $k$. That is, for some $3 \leq k_{0} \leq n$, we have

$$
\cdots \preccurlyeq \mathrm{BER}_{n, k_{0}-2} \preccurlyeq \mathrm{BER}_{n, k_{0}-1} \preccurlyeq \mathrm{BER}_{n, k_{0}} \succcurlyeq \mathrm{BER}_{n, k_{0}+1} \succcurlyeq \mathrm{BER}_{n, k_{0}+2} \succcurlyeq \cdots .
$$

Example 1. For $n=6$ and competence vector $\boldsymbol{p}=(0.98,0.8,0.8,0.7,0.7,0.5)$, we have $\mathrm{BER}_{6,3} \preccurlyeq \mathrm{BER}_{6,4} \preccurlyeq \mathrm{BER}_{6,5} \succcurlyeq \mathrm{BER}_{6,6}$. For $\boldsymbol{p}=(0.9,0.85,0.84,0.7,0.7,0.6)$, we have $\mathrm{BER}_{6,3} \preccurlyeq \mathrm{BER}_{6,4} \preccurlyeq \mathrm{BER}_{6,5} \preccurlyeq \mathrm{BER}_{6,6}$.

Theorem 3. For each $n$ and $p$, the sequence of decision rules $\mathrm{SER}_{n, k}$ is mound-shaped as a function of $k$. That is, for some $1 \leq k_{0} \leq n$, we have

$$
\cdots \preccurlyeq \operatorname{SER}_{n, k_{0}-4} \preccurlyeq \operatorname{SER}_{n, k_{0}-2} \preccurlyeq \operatorname{SER}_{n, k_{0}} \succcurlyeq \operatorname{SER}_{n, k_{0}+2} \succcurlyeq \operatorname{SER}_{n, k_{0}+4} \succcurlyeq \cdots .
$$

Theorems 2 and 3 might lead us to believe that the property of mound-shapedness is common to all sequences of rules that are monotonic in some sense. Somewhat surprisingly, the next theorem shows that the sequence of restricted majority rules behaves in a completely different way.

Theorem 4. For each $n$ and permutation $\left(\sigma_{1}, \sigma_{2}, \sigma_{3}, \ldots, \sigma_{m}\right)$ of the set $\{1,3,5, \ldots, 2 m-1\}$, where $m=\lfloor(n+1) / 2\rfloor$, there exists a vector of correctness probabilities for which

$$
\mathrm{RMR}_{\sigma_{1}} \succ \mathrm{RMR}_{\sigma_{2}} \succ \cdots \succ \mathrm{RMR}_{\sigma_{m}}
$$


In fact, it will become apparent from the proof that Theorem 4 also holds if some of the ' $\succ$ 's in (1) are replaced by ' $\sim$ '. For instance, for $n=11$, there exists a vector of correctness probabilities such that $\mathrm{RMR}_{5} \sim \mathrm{RMR}_{9} \succ \mathrm{RMR}_{3} \succ \mathrm{RMR}_{1} \sim \mathrm{RMR}_{11} \succ \mathrm{RMR}_{7}$.

\section{Proofs}

Proof of Theorem 3. Let us first show that if, for some $k$, we have $\mathrm{SER}_{n, k+2} \succcurlyeq \mathrm{SER}_{n, k}$ then $\mathrm{SER}_{n, k} \succcurlyeq \mathrm{SER}_{n, k-2}$. The voting profiles distinguishing between $\mathrm{SER}_{n, k+2}$ and $\mathrm{SER}_{n, k}$ are those profiles in which the first expert is supported by exactly $(n-k) / 2-1$ other experts. Similarly, voting profiles that distinguish between $\mathrm{SER}_{n, k}$ and $\mathrm{SER}_{n, k-2}$ are those in which the first expert is supported by exactly $(n-k) / 2$ other experts. Let $m=(n-k) / 2$. The condition $\mathrm{SER}_{n, k+2} \succcurlyeq \mathrm{SER}_{n, k}$ implies that

$$
p_{1} \sum_{E \in N_{\overline{1}, m-1}} \prod_{j \in E} p_{j} \prod_{j \in\{2, \ldots, n\} \backslash E} q_{j} \geq q_{1} \sum_{E \in N_{\overline{1}, n-m}} \prod_{j \in E} p_{j} \prod_{j \in\{2, \ldots, n\} \backslash E} q_{j},
$$

where $N_{\overline{1}, l}=\{E|E \subseteq\{2, \ldots, n\}| E \mid,=l\}$. This may be written in the form

$$
\alpha_{1} \sum_{i_{1}, \ldots, i_{m-1} \in\{2, \ldots, n\}} \alpha_{i_{1}} \cdots \alpha_{i_{m-1}} \geq \sum_{i_{1}, \ldots, i_{n-m} \in\{2, \ldots, n\}} \alpha_{i_{1}} \cdots \alpha_{i_{n-m}},
$$

or, equivalently,

$$
\alpha_{1} \sum_{i_{1}, \ldots, i_{m-1} \in\{2, \ldots, n\}} \alpha_{i_{1}} \cdots \alpha_{i_{m-1}} \geq \prod_{j=2}^{n} \alpha_{j} \sum_{i_{1}, \ldots, i_{m-1} \in\{2, \ldots, n\}} \frac{1}{\alpha_{i_{1}} \cdots \alpha_{i_{m-1}}} .
$$

We have to prove the analogue with $m$ instead of $m-1$ :

$$
\alpha_{1} \sum_{i_{1}, \ldots, i_{m} \in\{2, \ldots, n\}} \alpha_{i_{1}} \cdots \alpha_{i_{m}} \geq \prod_{j=2}^{n} \alpha_{j} \sum_{i_{1}, \ldots, i_{m} \in\{2, \ldots, n\}} \frac{1}{\alpha_{i_{1}} \cdots \alpha_{i_{m}}} .
$$

Observe that

$$
\alpha_{1} \sum_{i_{1}, \ldots, i_{m} \in\{2, \ldots, n\}} \alpha_{i_{1}} \ldots \alpha_{i_{m}}=\frac{1}{m} \alpha_{1} \sum_{i_{1}, \ldots, i_{m-1} \in\{2, \ldots, n\}}\left(\alpha_{i_{1}} \cdots \alpha_{i_{m-1}} \sum_{j \in\{2, \ldots, n\} \backslash\left\{i_{1}, \ldots, i_{m-1}\right\}} \alpha_{j}\right) .
$$

Since $\alpha_{j} \geq 1$ for $1 \leq j \leq n$, this implies that

$$
\alpha_{1} \sum_{i_{1}, \ldots, i_{m} \in\{2, \ldots, n\}} \alpha_{i_{1}} \cdots \alpha_{i_{m}} \geq \frac{n-m}{m} \alpha_{1} \sum_{i_{1}, \ldots, i_{m-1} \in\{2, \ldots, n\}} \alpha_{i_{1}} \cdots \alpha_{i_{m-1}}
$$

Similarly,

$$
\begin{aligned}
\sum_{i_{1}, \ldots, i_{m} \in\{2, \ldots, n\}} \frac{1}{\alpha_{i_{1}} \cdots \alpha_{i_{m}}} & =\frac{1}{m} \sum_{i_{1}, \ldots, i_{m-1} \in\{2, \ldots, n\}}\left(\frac{1}{\alpha_{i_{1}} \cdots \alpha_{i_{m-1}}} \sum_{j \in\{2, \ldots, n\} \backslash\left\{i_{1}, \ldots, i_{m-1}\right\}} \frac{1}{\alpha_{j}}\right) \\
& \leq \frac{n-m}{m} \sum_{i_{1}, \ldots, i_{m-1} \in\{2, \ldots, n\}} \frac{1}{\alpha_{i_{1}} \cdots \alpha_{i_{m-1}}} .
\end{aligned}
$$


Hence,

$$
\frac{n-m}{m} \prod_{j=2}^{n} \alpha_{j} \sum_{i_{1}, \ldots, i_{m-1} \in\{2, \ldots, n\}} \frac{1}{\alpha_{i_{1}} \cdots \alpha_{i_{m-1}}} \geq \prod_{j=2}^{n} \alpha_{j} \sum_{i_{1}, \ldots, i_{m} \in\{2, \ldots, n\}} \frac{1}{\alpha_{i_{1}} \cdots \alpha_{i_{m}}} .
$$

Combining (2), (4), and (5), we obtain (3).

In the same way we show that if, for some $k$, we have $\mathrm{SER}_{n, k-2} \succcurlyeq \mathrm{SER}_{n, k}$ then $\mathrm{SER}_{n, k} \succcurlyeq$ $\mathrm{SER}_{n, k+2}$.

Proof of Theorem 2. Let us first show that if, for some $k$, we have $\mathrm{BER}_{n, k+1} \succcurlyeq \mathrm{BER}_{n, k}$ then $\mathrm{BER}_{n, k} \succcurlyeq \mathrm{BER}_{n, k-1}$. The voting profiles distinguishing between $\mathrm{BER}_{n, k+1}$ and $\mathrm{BER}_{n, k}$ are those profiles in which the first and $(k+1)$ st expert are opposed by the experts $2, \ldots, k$. Similarly, the voting profiles that distinguish between $\mathrm{BER}_{n, k}$ and $\mathrm{BER}_{n, k-1}$ are those in which the first and the $k$ th expert are opposed by the experts $2, \ldots, k-1$. The inequality $\mathrm{BER}_{n, k+1} \succcurlyeq$ $\mathrm{BER}_{n, k}$ implies that

$$
p_{1} q_{2} \cdots q_{k} p_{k+1} \geq q_{1} p_{2} \cdots p_{k} q_{k+1} .
$$

This can be written in the form

$$
\alpha_{2} \cdots \alpha_{k} \leq \alpha_{1} \alpha_{k+1}
$$

Since $\alpha_{k} \geq \alpha_{k+1}$

$$
\alpha_{2} \cdots \alpha_{k-1} \leq \alpha_{1} \alpha_{k}
$$

which is equivalent to

$$
p_{1} q_{2} \cdots q_{k-1} p_{k}>q_{1} p_{2} \cdots p_{k-1} q_{k} .
$$

This proves that $\mathrm{BER}_{n, k} \succcurlyeq \mathrm{BER}_{n, k-1}$.

In the same way we show that if, for some $k$, we have $\mathrm{BER}_{n, k-1} \succcurlyeq \mathrm{BER}_{n, k}$ then $\mathrm{BER}_{n, k} \succcurlyeq$ $\mathrm{BER}_{n, k+1}$. The two implications prove the theorem.

Proof of Theorem 4. It suffices to deal with the case of odd $n$. In fact, if $n$ is even, and $\boldsymbol{p}=\left(p_{1}, p_{2}, \ldots, p_{n-1}\right)$ gives any required ordering of the $\mathrm{RMR}_{i} \mathrm{~s}$ for $n-1$ experts, then the vector $\boldsymbol{p}^{\prime}=\left(p_{1}, p_{2}, \ldots, p_{n}\right)$ gives the same ordering for $n$ experts for any $p_{n} \in\left(\frac{1}{2}, p_{n-1}\right)$. Define $v: \mathbb{R}^{m} \rightarrow \mathbb{R}^{n}$ by

$$
v\left(x_{1}, x_{2}, \ldots, x_{m}\right)=\left(x_{1}, x_{2}, x_{2}, x_{3}, x_{3}, \ldots, x_{m}, x_{m}\right), \quad\left(x_{1}, x_{2}, \ldots, x_{m}\right) \in \mathbb{R}^{m} .
$$

Also, define functions $f_{i}:[0,1]^{m} \rightarrow[0,1], 1 \leq i \leq m$, by

$$
f_{i}(\boldsymbol{r})=\pi\left(\mathrm{RMR}_{2 i-1}, v(\boldsymbol{r})\right), \quad \boldsymbol{r}=\left(r_{1}, r_{2}, \ldots, r_{m}\right) \in[0,1]^{m} .
$$

Consider the function $f:[0,1]^{m} \rightarrow[0,1]^{m}$ given by

$$
f(\boldsymbol{r})=\left(f_{1}(\boldsymbol{r}), f_{2}(\boldsymbol{r}), \ldots, f_{m}(\boldsymbol{r})\right), \quad \boldsymbol{r}=\left(r_{1}, r_{2}, \ldots, r_{m}\right) \in[0,1]^{m} .
$$

To prove the theorem, it suffices to show that if $\left(\sigma_{1}, \sigma_{2}, \ldots, \sigma_{m}\right)$ is any permutation of $\{1,2, \ldots, m\}$ then there exists a vector of probabilities $\boldsymbol{r}$ such that $f_{\sigma_{1}}(\boldsymbol{r})>f_{\sigma_{2}}(\boldsymbol{r})>\cdots>$ $f_{\sigma_{m}}(\boldsymbol{r})$.

Let $A=\left\{\left(r_{1}, r_{2}, \ldots, r_{m}\right): 1>r_{1}>r_{2}>\cdots>r_{m}>\frac{1}{2}\right\}$.

Let us first show that there exists a competence vector $\boldsymbol{r}^{0}=\left(r_{1}^{0}, r_{2}^{0}, \ldots, r_{m}^{0}\right) \in A$ such that $f_{1}\left(\boldsymbol{r}^{0}\right)=f_{2}\left(\boldsymbol{r}^{0}\right)=\cdots=f_{m}\left(\boldsymbol{r}^{0}\right)$. Choose an arbitrary $r_{1}^{0} \in\left(\frac{1}{2}, 1\right)$. Let $\alpha_{1}=r_{1}^{0} /(1-$ $\left.r_{1}^{0}\right)$. Next, let $\alpha_{2}=\alpha_{3}=\sqrt{\alpha_{1}}$ and $r_{2}^{0}=\alpha_{2} /\left(\alpha_{2}+1\right)$. In general, for $2 \leq i \leq m$, 
after $\alpha_{1}, \alpha_{2}, \alpha_{3}, \ldots, \alpha_{2 i-3}, r_{1}^{0}, r_{2}^{0}, \ldots, r_{i-1}^{0}$ have been defined, with $\alpha_{2 j}=\alpha_{2 j+1}$ and $r_{j}^{0}=$ $\alpha_{2 j-2} /\left(\alpha_{2 j-2}+1\right)$ for $1 \leq j \leq i-2$, we take

$$
\alpha_{2 i-2}=\alpha_{2 i-1}=\sqrt{\operatorname{CM}\left(\left(\alpha_{1}, \alpha_{2}, \ldots, \alpha_{2 i-3}\right)\right)}, \quad r_{i}^{0}=\frac{\alpha_{2 i-2}}{\alpha_{2 i-2}+1} .
$$

By induction we conclude, from Lemma 1 and Theorem 1, that $r_{1}^{0}>r_{2}^{0}>r_{3}^{0}>\cdots>r_{m}^{0}>\frac{1}{2}$ and that $f_{1}\left(\boldsymbol{r}^{0}\right)=f_{2}\left(\boldsymbol{r}^{0}\right)=\cdots=f_{m}\left(\boldsymbol{r}^{0}\right)$.

Next, let us show that $f$ is differentiable and its Jacobian matrix is invertible for any $\boldsymbol{r}=$ $\left(r_{1}, r_{2}, \ldots, r_{m}\right) \in A$. In fact, consider a group of experts with competence vector $v(\boldsymbol{r})$, where $\boldsymbol{r} \in A$. We have $f_{1}(\boldsymbol{r})=r_{1}$, so that $\partial f_{1}(\boldsymbol{r}) / \partial r_{1}=1>0$. For $2 \leq i \leq m$, denote by $P_{i, k}$ the probability that at least $k$ out of the $2 i-3$ most competent experts choose the correct alternative, and denote by $Q_{i, k}$ the probability that exactly $k$ of them do so. Then

$$
\begin{aligned}
f_{i}(\boldsymbol{r}) & =r_{i}^{2} P_{i, i-2}+2 r_{i}\left(1-r_{i}\right) P_{i, i-1}+\left(1-r_{i}\right)^{2} P_{i, i} \\
& =r_{i}^{2}\left(Q_{i, i-2}-Q_{i, i-1}\right)+2 r_{i} Q_{i, i-1}+P_{i, i}, \quad 2 \leq i \leq m .
\end{aligned}
$$

Since $\boldsymbol{r} \in A$, significantly small changes in $\boldsymbol{r}$ do not change the ranking of the experts, so that $P_{i, i}$ and each of the $Q_{i, j}$ s are polynomial in the variables $r_{1}, r_{2}, \ldots, r_{i-1}$. In particular, $f$ is differentiable on $A$. Now

$$
\frac{\partial f_{i}(\boldsymbol{r})}{\partial r_{i}}=2 r_{i}\left(Q_{i, i-2}-Q_{i, i-1}\right)+2 Q_{i, i-1}=2 r_{i} Q_{i, i-2}+2\left(1-r_{i}\right) Q_{i, i-1}, \quad 2 \leq i \leq m .
$$

As $\boldsymbol{r}$ belongs to $A$, sufficiently small changes in $r_{j}$ for $j>i$ do not effect $f_{i}(\boldsymbol{r})$, so that $\partial f_{i}(\boldsymbol{r}) / \partial r_{j}=0$ for $j>i$. Thus, the Jacobian matrix of $f$ is lower triangular with nonzero entries along the diagonal for every $r \in A$. In particular, it is invertible.

From Theorem 13.5 of Apostol (1974), it follows that $f$ is an open mapping on $A$. Since $f$ takes $\boldsymbol{r}^{0}$ to a point on the main diagonal of $\mathbb{R}^{m}$, this means that, for any permutation $\left(\sigma_{1}, \sigma_{2}, \ldots, \sigma_{m}\right)$ of $\{1,2, \ldots, m\}$, there exists a point $s=\left(s_{1}, s_{2}, \ldots, s_{m}\right) \in f(A)$ such that $s_{\sigma_{1}}>s_{\sigma_{2}}>\cdots>s_{\sigma_{m}}$. This completes the proof.

\section{Discussion and summary}

In this paper we considered three classes of decision rules-balanced expert rules, single expert rules, and restricted majority rules. These classes are 'similar' and 'different' in various aspects. In the first two families, the rules assign much of the decisional power to the most competent expert, whereas the restricted majority rules distribute it equally between all influential experts. In the context of our results, the first two families also share the same property of mound-shaped ranking, whereas in the family of restricted majority rules, any inner ranking is possible. On the other hand, both the family of single expert rules and the family of restricted majority rules contain the simple majority rule and the expert rule as the polar members. However, the two sequences of rules formed by the two families starting at the expert rule and ending at the simple majority rule have very different structures with respect to their ranking properties.

\section{Appendix A. All possible rankings for $\boldsymbol{n}=\mathbf{5}$}

1. $(1,0,0,0,0) \succ(3,1,1,1,1) \succ(2,1,1,1,0) \succ(3,2,2,1,1) \succ(2,2,1,1,1) \succ(1,1,1,1,1) \succ(1,1,1,0,0)$.

2. $(1,0,0,0,0) \succ(3,1,1,1,1) \succ(2,1,1,1,0) \succ(3,2,2,1,1) \succ(2,2,1,1,1) \succ(1,1,1,0,0) \succ(1,1,1,1,1)$. 
3. $(1,0,0,0,0) \succ(3,1,1,1,1) \succ(2,1,1,1,0) \succ(3,2,2,1,1) \succ(1,1,1,0,0) \succ(2,2,1,1,1) \succ(1,1,1,1,1)$.

4. $(3,1,1,1,1) \succ(1,0,0,0,0) \succ(2,1,1,1,0) \succ(3,2,2,1,1) \succ(2,2,1,1,1) \succ(1,1,1,1,1) \succ(1,1,1,0,0)$.

5. $(3,1,1,1,1) \succ(1,0,0,0,0) \succ(2,1,1,1,0) \succ(3,2,2,1,1) \succ(2,2,1,1,1) \succ(1,1,1,0,0) \succ(1,1,1,1,1)$.

6. $(3,1,1,1,1) \succ(1,0,0,0,0) \succ(2,1,1,1,0) \succ(3,2,2,1,1) \succ(1,1,1,0,0) \succ(2,2,1,1,1) \succ(1,1,1,1,1)$.

7. $(3,1,1,1,1) \succ(2,1,1,1,0) \succ(1,0,0,0,0) \succ(3,2,2,1,1) \succ(2,2,1,1,1) \succ(1,1,1,1,1) \succ(1,1,1,0,0)$.

8. $(3,1,1,1,1) \succ(2,1,1,1,0) \succ(1,0,0,0,0) \succ(3,2,2,1,1) \succ(2,2,1,1,1) \succ(1,1,1,0,0) \succ(1,1,1,1,1)$.

9. $(3,1,1,1,1) \succ(2,1,1,1,0) \succ(1,0,0,0,0) \succ(3,2,2,1,1) \succ(1,1,1,0,0) \succ(2,2,1,1,1) \succ(1,1,1,1,1)$.

10. $(3,1,1,1,1) \succ(2,1,1,1,0) \succ(3,2,2,1,1) \succ(1,0,0,0,0) \succ(2,2,1,1,1) \succ(1,1,1,1,1) \succ(1,1,1,0,0)$.

11. $(3,1,1,1,1) \succ(2,1,1,1,0) \succ(3,2,2,1,1) \succ(1,0,0,0,0) \succ(2,2,1,1,1) \succ(1,1,1,0,0) \succ(1,1,1,1,1)$.

12. $(3,1,1,1,1) \succ(2,1,1,1,0) \succ(3,2,2,1,1) \succ(1,0,0,0,0) \succ(1,1,1,0,0) \succ(2,2,1,1,1) \succ(1,1,1,1,1)$.

13. $(3,1,1,1,1) \succ(2,1,1,1,0) \succ(3,2,2,1,1) \succ(2,2,1,1,1) \succ(1,0,0,0,0) \succ(1,1,1,1,1) \succ(1,1,1,0,0)$.

14. $(3,1,1,1,1) \succ(2,1,1,1,0) \succ(3,2,2,1,1) \succ(2,2,1,1,1) \succ(1,0,0,0,0) \succ(1,1,1,0,0) \succ(1,1,1,1,1)$.

15. $(3,1,1,1,1) \succ(2,1,1,1,0) \succ(3,2,2,1,1) \succ(2,2,1,1,1) \succ(1,1,1,1,1) \succ(1,0,0,0,0) \succ(1,1,1,0,0)$.

16. $(2,1,1,1,0) \succ(3,1,1,1,1) \succ(1,0,0,0,0) \succ(3,2,2,1,1) \succ(2,2,1,1,1) \succ(1,1,1,1,1) \succ(1,1,1,0,0)$.

17. $(2,1,1,1,0) \succ(3,1,1,1,1) \succ(1,0,0,0,0) \succ(3,2,2,1,1) \succ(2,2,1,1,1) \succ(1,1,1,0,0) \succ(1,1,1,1,1)$.

18. $(2,1,1,1,0) \succ(3,1,1,1,1) \succ(1,0,0,0,0) \succ(3,2,2,1,1) \succ(1,1,1,0,0) \succ(2,2,1,1,1) \succ(1,1,1,1,1)$.

19. $(2,1,1,1,0) \succ(3,1,1,1,1) \succ(3,2,2,1,1) \succ(1,0,0,0,0) \succ(2,2,1,1,1) \succ(1,1,1,1,1) \succ(1,1,1,0,0)$.

20. $(2,1,1,1,0) \succ(3,1,1,1,1) \succ(3,2,2,1,1) \succ(1,0,0,0,0) \succ(2,2,1,1,1) \succ(1,1,1,0,0) \succ(1,1,1,1,1)$.

21. $(2,1,1,1,0) \succ(3,1,1,1,1) \succ(3,2,2,1,1) \succ(1,0,0,0,0) \succ(1,1,1,0,0) \succ(2,2,1,1,1) \succ(1,1,1,1,1)$.

22. $(2,1,1,1,0) \succ(3,1,1,1,1) \succ(3,2,2,1,1) \succ(2,2,1,1,1) \succ(1,0,0,0,0) \succ(1,1,1,1,1) \succ(1,1,1,0,0)$.

23. $(2,1,1,1,0) \succ(3,1,1,1,1) \succ(3,2,2,1,1) \succ(2,2,1,1,1) \succ(1,0,0,0,0) \succ(1,1,1,0,0) \succ(1,1,1,1,1)$.

24. $(2,1,1,1,0) \succ(3,1,1,1,1) \succ(3,2,2,1,1) \succ(2,2,1,1,1) \succ(1,1,1,1,1) \succ(1,0,0,0,0) \succ(1,1,1,0,0)$.

25. $(2,1,1,1,0) \succ(3,2,2,1,1) \succ(3,1,1,1,1) \succ(2,2,1,1,1) \succ(1,1,1,1,1) \succ(1,1,1,0,0) \succ(1,0,0,0,0)$.

26. $(2,1,1,1,0) \succ(3,2,2,1,1) \succ(3,1,1,1,1) \succ(2,2,1,1,1) \succ(1,1,1,0,0) \succ(1,0,0,0,0) \succ(1,1,1,1,1)$.

27. $(2,1,1,1,0) \succ(3,2,2,1,1) \succ(3,1,1,1,1) \succ(2,2,1,1,1) \succ(1,1,1,0,0) \succ(1,1,1,1,1) \succ(1,0,0,0,0)$.

28. $(2,1,1,1,0) \succ(3,2,2,1,1) \succ(3,1,1,1,1) \succ(1,1,1,0,0) \succ(1,0,0,0,0) \succ(2,2,1,1,1) \succ(1,1,1,1,1)$.

29. $(2,1,1,1,0) \succ(3,2,2,1,1) \succ(3,1,1,1,1) \succ(1,1,1,0,0) \succ(2,2,1,1,1) \succ(1,0,0,0,0) \succ(1,1,1,1,1)$.

30. $(2,1,1,1,0) \succ(3,2,2,1,1) \succ(3,1,1,1,1) \succ(1,1,1,0,0) \succ(2,2,1,1,1) \succ(1,1,1,1,1) \succ(1,0,0,0,0)$.

31. $(2,1,1,1,0) \succ(3,2,2,1,1) \succ(2,2,1,1,1) \succ(3,1,1,1,1) \succ(1,1,1,1,1) \succ(1,1,1,0,0) \succ(1,0,0,0,0)$.

32. $(2,1,1,1,0) \succ(3,2,2,1,1) \succ(2,2,1,1,1) \succ(3,1,1,1,1) \succ(1,1,1,0,0) \succ(1,0,0,0,0) \succ(1,1,1,1,1)$.

33. $(2,1,1,1,0) \succ(3,2,2,1,1) \succ(2,2,1,1,1) \succ(3,1,1,1,1) \succ(1,1,1,0,0) \succ(1,1,1,1,1) \succ(1,0,0,0,0)$.

34. $(2,1,1,1,0) \succ(3,2,2,1,1) \succ(2,2,1,1,1) \succ(1,1,1,1,1) \succ(3,1,1,1,1) \succ(1,1,1,0,0) \succ(1,0,0,0,0)$.

35. $(2,1,1,1,0) \succ(3,2,2,1,1) \succ(2,2,1,1,1) \succ(1,1,1,1,1) \succ(1,1,1,0,0) \succ(3,1,1,1,1) \succ(1,0,0,0,0)$.

36. $(2,1,1,1,0) \succ(3,2,2,1,1) \succ(2,2,1,1,1) \succ(1,1,1,0,0) \succ(3,1,1,1,1) \succ(1,0,0,0,0) \succ(1,1,1,1,1)$.

37. $(2,1,1,1,0) \succ(3,2,2,1,1) \succ(2,2,1,1,1) \succ(1,1,1,0,0) \succ(3,1,1,1,1) \succ(1,1,1,1,1) \succ(1,0,0,0,0)$.

38. $(2,1,1,1,0) \succ(3,2,2,1,1) \succ(2,2,1,1,1) \succ(1,1,1,0,0) \succ(1,1,1,1,1) \succ(3,1,1,1,1) \succ(1,0,0,0,0)$.

39. $(2,1,1,1,0) \succ(3,2,2,1,1) \succ(1,1,1,0,0) \succ(3,1,1,1,1) \succ(1,0,0,0,0) \succ(2,2,1,1,1) \succ(1,1,1,1,1)$. 
40. $(2,1,1,1,0) \succ(3,2,2,1,1) \succ(1,1,1,0,0) \succ(3,1,1,1,1) \succ(2,2,1,1,1) \succ(1,0,0,0,0) \succ(1,1,1,1,1)$. 41. $(2,1,1,1,0) \succ(3,2,2,1,1) \succ(1,1,1,0,0) \succ(3,1,1,1,1) \succ(2,2,1,1,1) \succ(1,1,1,1,1) \succ(1,0,0,0,0)$. 42. $(2,1,1,1,0) \succ(3,2,2,1,1) \succ(1,1,1,0,0) \succ(2,2,1,1,1) \succ(3,1,1,1,1) \succ(1,0,0,0,0) \succ(1,1,1,1,1)$. 43. $(2,1,1,1,0) \succ(3,2,2,1,1) \succ(1,1,1,0,0) \succ(2,2,1,1,1) \succ(3,1,1,1,1) \succ(1,1,1,1,1) \succ(1,0,0,0,0)$. 44. $(2,1,1,1,0) \succ(3,2,2,1,1) \succ(1,1,1,0,0) \succ(2,2,1,1,1) \succ(1,1,1,1,1) \succ(3,1,1,1,1) \succ(1,0,0,0,0)$. 45. $(3,2,2,1,1) \succ(2,1,1,1,0) \succ(3,1,1,1,1) \succ(2,2,1,1,1) \succ(1,1,1,1,1) \succ(1,1,1,0,0) \succ(1,0,0,0,0)$. 46. $(3,2,2,1,1) \succ(2,1,1,1,0) \succ(3,1,1,1,1) \succ(2,2,1,1,1) \succ(1,1,1,0,0) \succ(1,0,0,0,0) \succ(1,1,1,1,1)$. 47. $(3,2,2,1,1) \succ(2,1,1,1,0) \succ(3,1,1,1,1) \succ(2,2,1,1,1) \succ(1,1,1,0,0) \succ(1,1,1,1,1) \succ(1,0,0,0,0)$. 48. $(3,2,2,1,1) \succ(2,1,1,1,0) \succ(3,1,1,1,1) \succ(1,1,1,0,0) \succ(1,0,0,0,0) \succ(2,2,1,1,1) \succ(1,1,1,1,1)$. 49. $(3,2,2,1,1) \succ(2,1,1,1,0) \succ(3,1,1,1,1) \succ(1,1,1,0,0) \succ(2,2,1,1,1) \succ(1,0,0,0,0) \succ(1,1,1,1,1)$. 50. $(3,2,2,1,1) \succ(2,1,1,1,0) \succ(3,1,1,1,1) \succ(1,1,1,0,0) \succ(2,2,1,1,1) \succ(1,1,1,1,1) \succ(1,0,0,0,0)$. 51. $(3,2,2,1,1) \succ(2,1,1,1,0) \succ(2,2,1,1,1) \succ(3,1,1,1,1) \succ(1,1,1,1,1) \succ(1,1,1,0,0) \succ(1,0,0,0,0)$. 52. $(3,2,2,1,1) \succ(2,1,1,1,0) \succ(2,2,1,1,1) \succ(3,1,1,1,1) \succ(1,1,1,0,0) \succ(1,0,0,0,0) \succ(1,1,1,1,1)$. 53. $(3,2,2,1,1) \succ(2,1,1,1,0) \succ(2,2,1,1,1) \succ(3,1,1,1,1) \succ(1,1,1,0,0) \succ(1,1,1,1,1) \succ(1,0,0,0,0)$. 54. $(3,2,2,1,1) \succ(2,1,1,1,0) \succ(2,2,1,1,1) \succ(1,1,1,1,1) \succ(3,1,1,1,1) \succ(1,1,1,0,0) \succ(1,0,0,0,0)$. 55. $(3,2,2,1,1) \succ(2,1,1,1,0) \succ(2,2,1,1,1) \succ(1,1,1,1,1) \succ(1,1,1,0,0) \succ(3,1,1,1,1) \succ(1,0,0,0,0)$. 56. $(3,2,2,1,1) \succ(2,1,1,1,0) \succ(2,2,1,1,1) \succ(1,1,1,0,0) \succ(3,1,1,1,1) \succ(1,0,0,0,0) \succ(1,1,1,1,1)$. 57. $(3,2,2,1,1) \succ(2,1,1,1,0) \succ(2,2,1,1,1) \succ(1,1,1,0,0) \succ(3,1,1,1,1) \succ(1,1,1,1,1) \succ(1,0,0,0,0)$. 58. $(3,2,2,1,1) \succ(2,1,1,1,0) \succ(2,2,1,1,1) \succ(1,1,1,0,0) \succ(1,1,1,1,1) \succ(3,1,1,1,1) \succ(1,0,0,0,0)$. 59. $(3,2,2,1,1) \succ(2,1,1,1,0) \succ(1,1,1,0,0) \succ(3,1,1,1,1) \succ(1,0,0,0,0) \succ(2,2,1,1,1) \succ(1,1,1,1,1)$. 60. $(3,2,2,1,1) \succ(2,1,1,1,0) \succ(1,1,1,0,0) \succ(3,1,1,1,1) \succ(2,2,1,1,1) \succ(1,0,0,0,0) \succ(1,1,1,1,1)$. 61. $(3,2,2,1,1) \succ(2,1,1,1,0) \succ(1,1,1,0,0) \succ(3,1,1,1,1) \succ(2,2,1,1,1) \succ(1,1,1,1,1) \succ(1,0,0,0,0)$. 62. $(3,2,2,1,1) \succ(2,1,1,1,0) \succ(1,1,1,0,0) \succ(2,2,1,1,1) \succ(3,1,1,1,1) \succ(1,0,0,0,0) \succ(1,1,1,1,1)$. 63. $(3,2,2,1,1) \succ(2,1,1,1,0) \succ(1,1,1,0,0) \succ(2,2,1,1,1) \succ(3,1,1,1,1) \succ(1,1,1,1,1) \succ(1,0,0,0,0)$. 64. $(3,2,2,1,1) \succ(2,1,1,1,0) \succ(1,1,1,0,0) \succ(2,2,1,1,1) \succ(1,1,1,1,1) \succ(3,1,1,1,1) \succ(1,0,0,0,0)$. 65. $(3,2,2,1,1) \succ(2,2,1,1,1) \succ(2,1,1,1,0) \succ(3,1,1,1,1) \succ(1,1,1,1,1) \succ(1,1,1,0,0) \succ(1,0,0,0,0)$. 66. $(3,2,2,1,1) \succ(2,2,1,1,1) \succ(2,1,1,1,0) \succ(3,1,1,1,1) \succ(1,1,1,0,0) \succ(1,0,0,0,0) \succ(1,1,1,1,1)$. 67. $(3,2,2,1,1) \succ(2,2,1,1,1) \succ(2,1,1,1,0) \succ(3,1,1,1,1) \succ(1,1,1,0,0) \succ(1,1,1,1,1) \succ(1,0,0,0,0)$. 68. $(3,2,2,1,1) \succ(2,2,1,1,1) \succ(2,1,1,1,0) \succ(1,1,1,1,1) \succ(3,1,1,1,1) \succ(1,1,1,0,0) \succ(1,0,0,0,0)$. 69. $(3,2,2,1,1) \succ(2,2,1,1,1) \succ(2,1,1,1,0) \succ(1,1,1,1,1) \succ(1,1,1,0,0) \succ(3,1,1,1,1) \succ(1,0,0,0,0)$. 70. $(3,2,2,1,1) \succ(2,2,1,1,1) \succ(2,1,1,1,0) \succ(1,1,1,0,0) \succ(3,1,1,1,1) \succ(1,0,0,0,0) \succ(1,1,1,1,1)$. 71. $(3,2,2,1,1) \succ(2,2,1,1,1) \succ(2,1,1,1,0) \succ(1,1,1,0,0) \succ(3,1,1,1,1) \succ(1,1,1,1,1) \succ(1,0,0,0,0)$. 72. $(3,2,2,1,1) \succ(2,2,1,1,1) \succ(2,1,1,1,0) \succ(1,1,1,0,0) \succ(1,1,1,1,1) \succ(3,1,1,1,1) \succ(1,0,0,0,0)$. 73. $(3,2,2,1,1) \succ(2,2,1,1,1) \succ(1,1,1,1,1) \succ(2,1,1,1,0) \succ(3,1,1,1,1) \succ(1,1,1,0,0) \succ(1,0,0,0,0)$. 74. $(3,2,2,1,1) \succ(2,2,1,1,1) \succ(1,1,1,1,1) \succ(2,1,1,1,0) \succ(1,1,1,0,0) \succ(3,1,1,1,1) \succ(1,0,0,0,0)$. 75. $(3,2,2,1,1) \succ(2,2,1,1,1) \succ(1,1,1,1,1) \succ(1,1,1,0,0) \succ(2,1,1,1,0) \succ(3,1,1,1,1) \succ(1,0,0,0,0)$. 76. $(3,2,2,1,1) \succ(2,2,1,1,1) \succ(1,1,1,0,0) \succ(2,1,1,1,0) \succ(3,1,1,1,1) \succ(1,0,0,0,0) \succ(1,1,1,1,1)$. 
77. $(3,2,2,1,1) \succ(2,2,1,1,1) \succ(1,1,1,0,0) \succ(2,1,1,1,0) \succ(3,1,1,1,1) \succ(1,1,1,1,1) \succ(1,0,0,0,0)$. 78. $(3,2,2,1,1) \succ(2,2,1,1,1) \succ(1,1,1,0,0) \succ(2,1,1,1,0) \succ(1,1,1,1,1) \succ(3,1,1,1,1) \succ(1,0,0,0,0)$. 79. $(3,2,2,1,1) \succ(2,2,1,1,1) \succ(1,1,1,0,0) \succ(1,1,1,1,1) \succ(2,1,1,1,0) \succ(3,1,1,1,1) \succ(1,0,0,0,0)$. 80. $(3,2,2,1,1) \succ(1,1,1,0,0) \succ(2,1,1,1,0) \succ(3,1,1,1,1) \succ(1,0,0,0,0) \succ(2,2,1,1,1) \succ(1,1,1,1,1)$. 81. $(3,2,2,1,1) \succ(1,1,1,0,0) \succ(2,1,1,1,0) \succ(3,1,1,1,1) \succ(2,2,1,1,1) \succ(1,0,0,0,0) \succ(1,1,1,1,1)$. 82. $(3,2,2,1,1) \succ(1,1,1,0,0) \succ(2,1,1,1,0) \succ(3,1,1,1,1) \succ(2,2,1,1,1) \succ(1,1,1,1,1) \succ(1,0,0,0,0)$. 83. $(3,2,2,1,1) \succ(1,1,1,0,0) \succ(2,1,1,1,0) \succ(2,2,1,1,1) \succ(3,1,1,1,1) \succ(1,0,0,0,0) \succ(1,1,1,1,1)$. 84. $(3,2,2,1,1) \succ(1,1,1,0,0) \succ(2,1,1,1,0) \succ(2,2,1,1,1) \succ(3,1,1,1,1) \succ(1,1,1,1,1) \succ(1,0,0,0,0)$. 85. $(3,2,2,1,1) \succ(1,1,1,0,0) \succ(2,1,1,1,0) \succ(2,2,1,1,1) \succ(1,1,1,1,1) \succ(3,1,1,1,1) \succ(1,0,0,0,0)$. 86. $(3,2,2,1,1) \succ(1,1,1,0,0) \succ(2,2,1,1,1) \succ(2,1,1,1,0) \succ(3,1,1,1,1) \succ(1,0,0,0,0) \succ(1,1,1,1,1)$. 87. $(3,2,2,1,1) \succ(1,1,1,0,0) \succ(2,2,1,1,1) \succ(2,1,1,1,0) \succ(3,1,1,1,1) \succ(1,1,1,1,1) \succ(1,0,0,0,0)$. 88. $(3,2,2,1,1) \succ(1,1,1,0,0) \succ(2,2,1,1,1) \succ(2,1,1,1,0) \succ(1,1,1,1,1) \succ(3,1,1,1,1) \succ(1,0,0,0,0)$. 89. $(3,2,2,1,1) \succ(1,1,1,0,0) \succ(2,2,1,1,1) \succ(1,1,1,1,1) \succ(2,1,1,1,0) \succ(3,1,1,1,1) \succ(1,0,0,0,0)$. 90. $(2,2,1,1,1) \succ(3,2,2,1,1) \succ(2,1,1,1,0) \succ(3,1,1,1,1) \succ(1,1,1,1,1) \succ(1,1,1,0,0) \succ(1,0,0,0,0)$. 91. $(2,2,1,1,1) \succ(3,2,2,1,1) \succ(2,1,1,1,0) \succ(3,1,1,1,1) \succ(1,1,1,0,0) \succ(1,0,0,0,0) \succ(1,1,1,1,1)$. 92. $(2,2,1,1,1) \succ(3,2,2,1,1) \succ(2,1,1,1,0) \succ(3,1,1,1,1) \succ(1,1,1,0,0) \succ(1,1,1,1,1) \succ(1,0,0,0,0)$. 93. $(2,2,1,1,1) \succ(3,2,2,1,1) \succ(2,1,1,1,0) \succ(1,1,1,1,1) \succ(3,1,1,1,1) \succ(1,1,1,0,0) \succ(1,0,0,0,0)$. 94. $(2,2,1,1,1) \succ(3,2,2,1,1) \succ(2,1,1,1,0) \succ(1,1,1,1,1) \succ(1,1,1,0,0) \succ(3,1,1,1,1) \succ(1,0,0,0,0)$. 95. $(2,2,1,1,1) \succ(3,2,2,1,1) \succ(2,1,1,1,0) \succ(1,1,1,0,0) \succ(3,1,1,1,1) \succ(1,0,0,0,0) \succ(1,1,1,1,1)$. 96. $(2,2,1,1,1) \succ(3,2,2,1,1) \succ(2,1,1,1,0) \succ(1,1,1,0,0) \succ(3,1,1,1,1) \succ(1,1,1,1,1) \succ(1,0,0,0,0)$. 97. $(2,2,1,1,1) \succ(3,2,2,1,1) \succ(2,1,1,1,0) \succ(1,1,1,0,0) \succ(1,1,1,1,1) \succ(3,1,1,1,1) \succ(1,0,0,0,0)$. 98. $(2,2,1,1,1) \succ(3,2,2,1,1) \succ(1,1,1,1,1) \succ(2,1,1,1,0) \succ(3,1,1,1,1) \succ(1,1,1,0,0) \succ(1,0,0,0,0)$. 99. $(2,2,1,1,1) \succ(3,2,2,1,1) \succ(1,1,1,1,1) \succ(2,1,1,1,0) \succ(1,1,1,0,0) \succ(3,1,1,1,1) \succ(1,0,0,0,0)$. 100. $(2,2,1,1,1) \succ(3,2,2,1,1) \succ(1,1,1,1,1) \succ(1,1,1,0,0) \succ(2,1,1,1,0) \succ(3,1,1,1,1) \succ(1,0,0,0,0)$. 101. $(2,2,1,1,1) \succ(3,2,2,1,1) \succ(1,1,1,0,0) \succ(2,1,1,1,0) \succ(3,1,1,1,1) \succ(1,0,0,0,0) \succ(1,1,1,1,1)$. 102. $(2,2,1,1,1) \succ(3,2,2,1,1) \succ(1,1,1,0,0) \succ(2,1,1,1,0) \succ(3,1,1,1,1) \succ(1,1,1,1,1) \succ(1,0,0,0,0)$. 103. $(2,2,1,1,1) \succ(3,2,2,1,1) \succ(1,1,1,0,0) \succ(2,1,1,1,0) \succ(1,1,1,1,1) \succ(3,1,1,1,1) \succ(1,0,0,0,0)$. 104. $(2,2,1,1,1) \succ(3,2,2,1,1) \succ(1,1,1,0,0) \succ(1,1,1,1,1) \succ(2,1,1,1,0) \succ(3,1,1,1,1) \succ(1,0,0,0,0)$. 105. $(2,2,1,1,1) \succ(1,1,1,1,1) \succ(3,2,2,1,1) \succ(2,1,1,1,0) \succ(3,1,1,1,1) \succ(1,1,1,0,0) \succ(1,0,0,0,0)$. 106. $(2,2,1,1,1) \succ(1,1,1,1,1) \succ(3,2,2,1,1) \succ(2,1,1,1,0) \succ(1,1,1,0,0) \succ(3,1,1,1,1) \succ(1,0,0,0,0)$. 107. $(2,2,1,1,1) \succ(1,1,1,1,1) \succ(3,2,2,1,1) \succ(1,1,1,0,0) \succ(2,1,1,1,0) \succ(3,1,1,1,1) \succ(1,0,0,0,0)$. 108. $(1,1,1,1,1) \succ(2,2,1,1,1) \succ(3,2,2,1,1) \succ(2,1,1,1,0) \succ(3,1,1,1,1) \succ(1,1,1,0,0) \succ(1,0,0,0,0)$. 109. $(1,1,1,1,1) \succ(2,2,1,1,1) \succ(3,2,2,1,1) \succ(2,1,1,1,0) \succ(1,1,1,0,0) \succ(3,1,1,1,1) \succ(1,0,0,0,0)$. 110. $(1,1,1,1,1) \succ(2,2,1,1,1) \succ(3,2,2,1,1) \succ(1,1,1,0,0) \succ(2,1,1,1,0) \succ(3,1,1,1,1) \succ(1,0,0,0,0)$. 111. $(1,1,1,0,0) \succ(3,2,2,1,1) \succ(2,1,1,1,0) \succ(3,1,1,1,1) \succ(1,0,0,0,0) \succ(2,2,1,1,1) \succ(1,1,1,1,1)$. 112. $(1,1,1,0,0) \succ(3,2,2,1,1) \succ(2,1,1,1,0) \succ(3,1,1,1,1) \succ(2,2,1,1,1) \succ(1,0,0,0,0) \succ(1,1,1,1,1)$. 113. $(1,1,1,0,0) \succ(3,2,2,1,1) \succ(2,1,1,1,0) \succ(3,1,1,1,1) \succ(2,2,1,1,1) \succ(1,1,1,1,1) \succ(1,0,0,0,0)$. 
114. $(1,1,1,0,0) \succ(3,2,2,1,1) \succ(2,1,1,1,0) \succ(2,2,1,1,1) \succ(3,1,1,1,1) \succ(1,0,0,0,0) \succ(1,1,1,1,1)$.

115. $(1,1,1,0,0) \succ(3,2,2,1,1) \succ(2,1,1,1,0) \succ(2,2,1,1,1) \succ(3,1,1,1,1) \succ(1,1,1,1,1) \succ(1,0,0,0,0)$.

116. $(1,1,1,0,0) \succ(3,2,2,1,1) \succ(2,1,1,1,0) \succ(2,2,1,1,1) \succ(1,1,1,1,1) \succ(3,1,1,1,1) \succ(1,0,0,0,0)$.

117. $(1,1,1,0,0) \succ(3,2,2,1,1) \succ(2,2,1,1,1) \succ(2,1,1,1,0) \succ(3,1,1,1,1) \succ(1,0,0,0,0) \succ(1,1,1,1,1)$.

118. $(1,1,1,0,0) \succ(3,2,2,1,1) \succ(2,2,1,1,1) \succ(2,1,1,1,0) \succ(3,1,1,1,1) \succ(1,1,1,1,1) \succ(1,0,0,0,0)$.

119. $(1,1,1,0,0) \succ(3,2,2,1,1) \succ(2,2,1,1,1) \succ(2,1,1,1,0) \succ(1,1,1,1,1) \succ(3,1,1,1,1) \succ(1,0,0,0,0)$.

120. $(1,1,1,0,0) \succ(3,2,2,1,1) \succ(2,2,1,1,1) \succ(1,1,1,1,1) \succ(2,1,1,1,0) \succ(3,1,1,1,1) \succ(1,0,0,0,0)$.

\section{References}

Apostol, T. M. (1974). Mathematical Analysis, 2nd edn. Addison-Wesley, Reading, MA.

Berend, D. And SAPIR, L. (2003). Between the expert and majority rules. Adv. Appl. Prob. 35, 941-960.

De Condorcet, N. C. (1785). Essai sur l'application de l'analyse à la probabilité des décisions rendues á la pluralité des voix. Paris.

Gradstein, M. And NitZan, S. (1986). Performance evaluation of some special classes of weighted majority rules. Math. Soc. Sci. 12, 31-46.

Isbell, J. (1959). On the enumeration of majority games. Math. Tables Aids Comput. 13, 21-28.

Karotkin, D. (1993). Inferiority of restricted majority decision rules. Public Choice 77, 249-258.

KAROTKIN, D. (1998). The network of weighted majority rules and weighted majority games. Games Econom. Behavior 22, 299-315.

KarotKin, D. AND Schaps, M. (2003). The network of weighted majority rules and its geometric realizations. Games Econom. Behavior 42, 75-90.

KarotKin, D., NitZan, S. AND PARoush, J. (1988). The essential ranking of decision rules in small panels of experts. Theory Decision 24, 253-268.

Muroga, S., Toda, I. And Kondo, M. (1967). Enumeration of threshold functions of eight variables. Tech. Rep. 245, Department of Computer Science, University of Illinois.

Nitzan, S. ANd Paroush, J. (1982). Optimal decision rules in uncertain dichotomous choice situations. Internat. Econom. Rev. 23, 289-297.

NitZan, S. And Paroush, J. (1984). A general theorem and eight corollaries in search of a correct decision. Theory Decision 17, 211-220.

Nitzan, S. And Paroush, J. (1985). Collective Decision Making: An Economic Outlook. Cambridge University Press.

SAPIR, L. (2005). The mean competence of decision makers as a criterion for small changes. Unpublished manuscript. Von Neumann, J. and Morgenstern, O. (1944). Theory of Games and Economic Behavior. Princeton University Press. 